\title{
Perineal hibernoma: a case report and literature review
}

\section{Hibernoma perineal: presentación de un caso clínico y revisión de la literatura}

\author{
DÓscar Legido-Gómez,1* (iD Saúl Rico-Marco, ${ }^{1}$ (DInmaculada Díaz de Mera Sánchez-Migallón, ${ }^{1}$ \\ (DLaura Heeraíz-Raya, ${ }^{1}$ iD Germán Fliquete-Serrano, ${ }^{1}$ DPedro Carrión-López, ${ }^{1}$ \\ (iD) Antonio Santiago Salinas-Sánchez. ${ }^{1}$
}

Key words:

Hibernoma, Brown fat, Lipomatous neoplasia

Correspondencia: * Óscar Legido Gómez,

Dirección: Complejo Hospitalario Universitario de Albacete, Avenida Ramón y Cajal 27A $3^{\circ} \mathrm{B}$, Albacate, España. Correo electrónico: oscarlg9@ hotmail.es

\section{Abstract}

Clinical case: The present article describes the case of a 48-yearold male patient with no history of interest referred from primary care to evaluate a left perineal tumour occasionally painful. Radiological findings reveal a fatty lesion whose suspected diagnosis is hibernoma being confirmed once the histopathological examination is made.

Relevance: Hibernoma is frequently mistaken for lipoma or liposarcoma due to its clinical and radiological features, being crucial the differentiation to avoid potentially iatrogenic therapies.

Conclusion: Hibernoma is a rare, slow-growing and painless benign neoplasia originated from brown fat. Although it can be suspected according to diagnostic imaging, the definitive diagnosis is histological and the treatment of choice consists of a complete resection. There are no reported cases of recurrence or metastases at the long-term follow-up.

Citación: Legido-Gómez O, Rico-Marco, S, Díaz de Mera Sánchez-Migallón I, Heeraíz-Raya L., Fliquete-Serrano G., Carrión-López P., et al. Hibernoma perineal: presentación de un caso clínico y revisión de la literatura. Rev Mex Urol. 2021;81(2):pp 1-7

\section{${ }^{1}$ Complejo Hospitalario Universitario de Albacete, Albacete, España}

Recibido: 11 de junio de 2020 Aceptado: 30 de abril de 2021

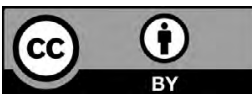




\section{Hibernoma perineal: presentación de un caso clínico y revisión de la literatura. Legido-Gómez Ó., et al.}

Palabras clave: Hibernoma, grasa parda, neoplasia de tejido adiposo. $\quad$ a largo plazo.

\section{Resumen}

Descripción del caso clínico: Se expone el caso de un paciente varón de 48 años sin antecedentes de interés que acude derivado de atención primaria para el estudio de una tumoración perineal izquierda que le produce dolor ocasional. Los hallazgos de los estudios radiológicos practicados revelan una lesión de estirpe grasa con sospecha de hibernoma, confirmándose anatomopatológicamente el diagnóstico.

Relevancia: El hibernoma es frecuentemente confundido con un lipoma o liposarcoma por sus similitudes clínicas y radiológicas, por lo que es crucial su diferenciación para evitar la aplicación de tratamientos potencialmente iatrogénicos.

Conclusión: El hibernoma es una neoplasia benigna poco frecuente originada en tejido graso pardo, de crecimiento lento e indoloro. Aunque pueda ser sospechado en base a los resultados de las pruebas de imagen, el diagnóstico definitivo es histológico y el tratamiento de elección es la extirpación completa. No se han descrito casos de recurrencia ni metástasis en el seguimiento

\section{Antecedentes}

El hibernoma es una neoplasia benigna que tiene su origen en la grasa parda, representando el 1.6\% de los tumores adipocitarios benignos y un $1.1 \%$ del total de este tipo de neoplasias. ${ }^{(1)}$

Este tumor fue primeramente descrito como "pseudolipoma" por Merkel en 1906 y el término "hibernoma" fue acuñado en 1914 por Grey debido a su parecido morfológico a la grasa parda objetivada en animales que hibernan. ${ }^{(2)}$

El tejido adiposo se presenta en la mayoría de los mamíferos en dos variedades: grasa blanca y grasa parda, siendo la primera su componente mayoritario. La grasa parda constituye un $5 \%$ del peso corporal en neonatos y progre- sivamente va disminuyendo con el crecimiento, persistiendo únicamente en determinadas localizaciones corporales en el adulto (cuello, mediastino, retroperitoneo y axila). Su función principal es proporcionar aislamiento contra la pérdida de calor, aunque también cumple una función metabólica al garantizar el almacenamiento de reservas energéticas. ${ }^{(3)}$

El hecho de que en la literatura existan un reducido número de casos junto con la ausencia de artículos que describan hibernomas de localización perineal reflejan la rareza de esta neoplasia y motivan por tanto la publicación de este caso clínico. 


\section{Caso clínico}

Varón de 48 años sin antecedentes de interés derivado de atención primaria para el estudio de una tumoración perineal inferior y lateral a hemiescroto izquierdo. El paciente refería dolor ocasional, impidiéndole la realización de sus actividades diarias. A la exploración física, se objetivaba una lesión móvil de consistencia blanda, elástica, de morfología redondeada y ligeramente dolorosa a la palpación profunda en cuadrante superior izquierdo de periné. La ecografía de la tumoración revelaba una imagen nodular de aumento de partes blandas a expensas de tejido subcutáneo, de límites mal definidos y con una ecogenicidad heterogénea sin aumento de flujo en Doppler color.

Una tomografía computarizada (TC) abdominopélvica con contraste mostró una lesión sólida de $6,1 \mathrm{~cm}$ de diámetro anteroposterior $\mathrm{x}$ $3 \mathrm{~cm}$ de diámetro transverso de densidad grasa, ligeramente más hiperdensa que la circundante con vascularización en su interior. (Figura 1)

Figura 1. TC. Lesión sólida de $6 \times 3 \mathrm{~cm}$ de densidad grasa más hiperdensa que el tejido adiposo circundante

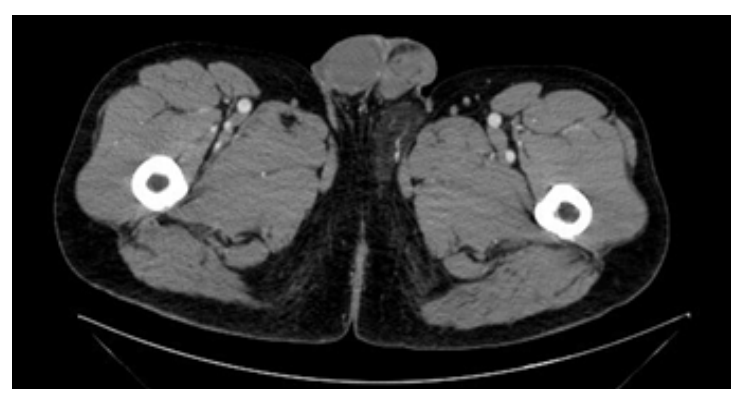

Para su mejor caracterización fue practicada una resonancia magnética (RM), visualizándose la tumoración descrita levemente hipointensa en $\mathrm{T} 1$ con respecto a la grasa locorregional, con contenido graso en su interior, aunque sin suprimirlo completamente en secuencia selectiva SPAIR pero sí en la DIXON (Figura 2), planteando el diagnóstico radiológico de hibernoma como primera posibilidad. No se visualizaron adenopatías ni lesiones óseas.

Figura 2. RM. Primera imagen, secuencia T1 que muestra una lesión hipointensa con respecto a la grasa locorregional. En el centro, secuencia T2 con técnica de supresión grasa SPAIR con supresión incompleta del contenido graso. Última, secuencia T2 con técnica DIXON que suprime completamente la grasa lesional
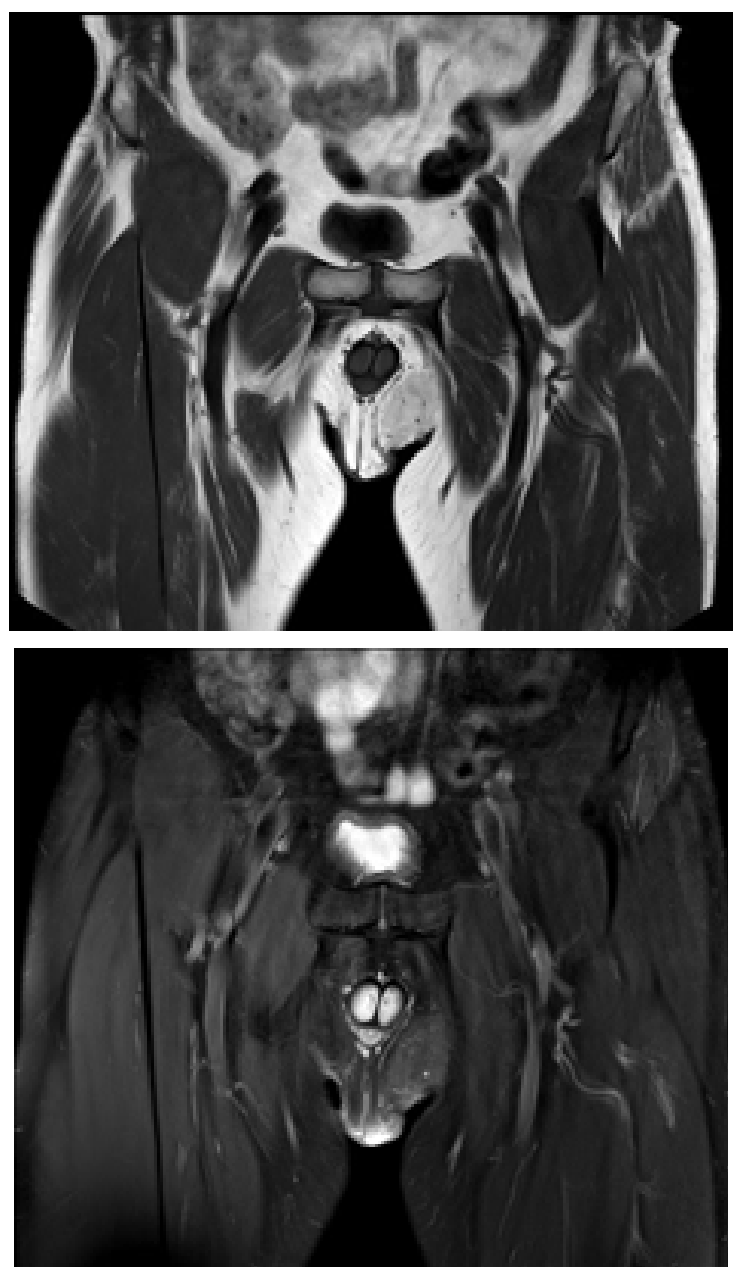


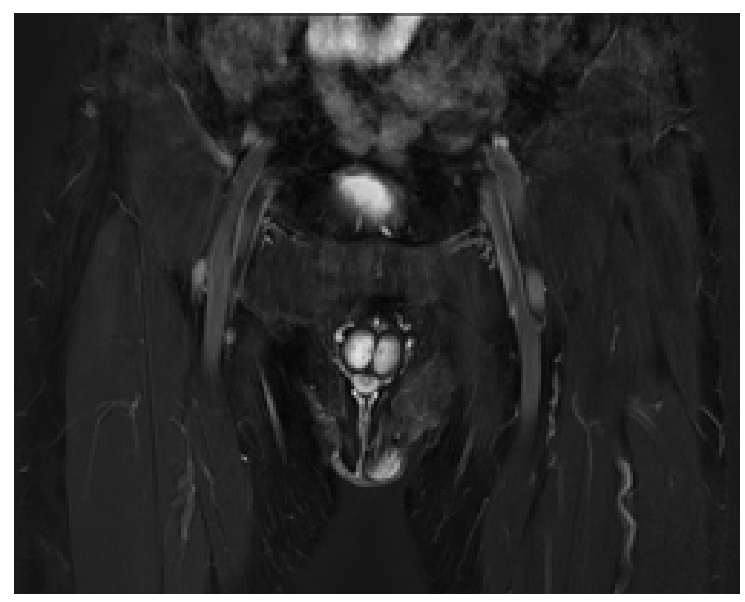

El paciente fue intervenido practicándose exéresis completa de la lesión. Su análisis anatomopatológico confirmó el diagnóstico de sospecha: macroscópicamente se trataba de una masa grasa de aspecto parduzco que al examen microscópico presentaba adipocitos maduros monovacuolados de tipo habitual (núcleo celular excéntrico) junto con adipocitos con citoplasma granular eosinofílico y núcleo centrado presentes en tejido graso pardo organizados en lóbulos, sin mostrar focos de atipias ni mitosis. (Figuras 3 y 4 )

Figura 3: Análisis microscópico en 10x. Tinción de hematoxilina-eosina. Se objetivan dos poblaciones celulares de adipocitos correspondientes a grasa blanca y grasa parda

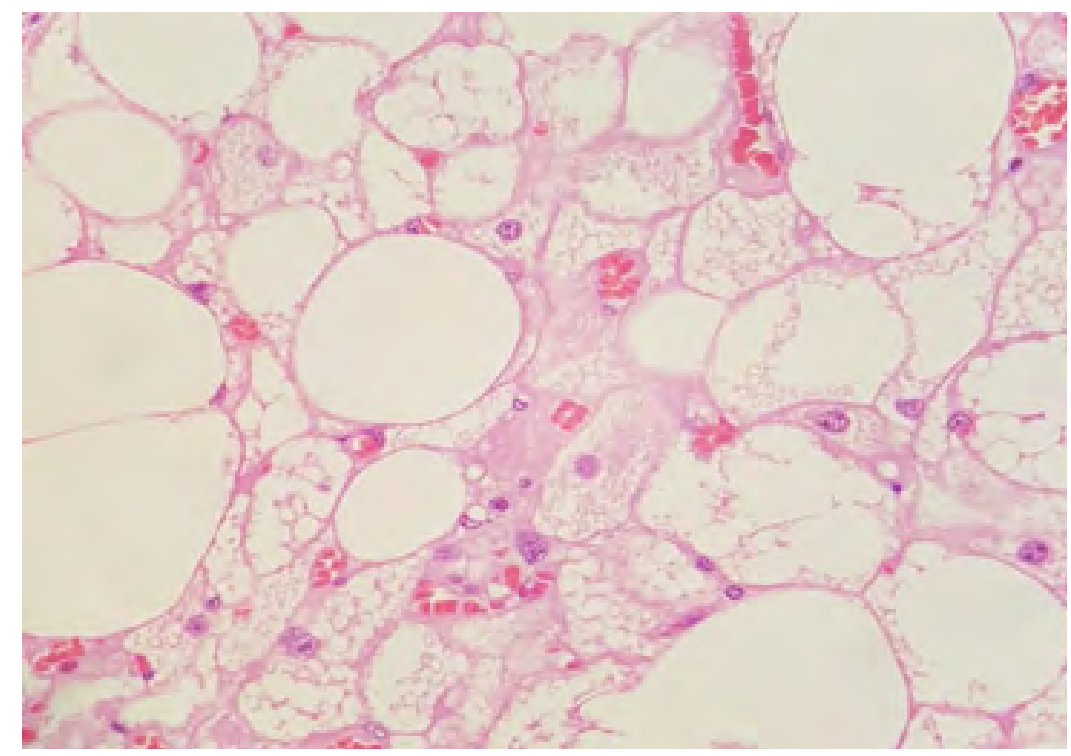


Figura 4: Análisis microscópico en 40x. Tinción de hematoxilina-eosina. Los adipocitos de grasa blanca son maduros, monovacuolados y con núcleo celular periférico (flecha continua). Los de tejido graso pardo presentan citoplasma granular eosinófilo y núcleo en el centro de la célula (flecha discontinua). No se observan focos de atipias ni mitosis

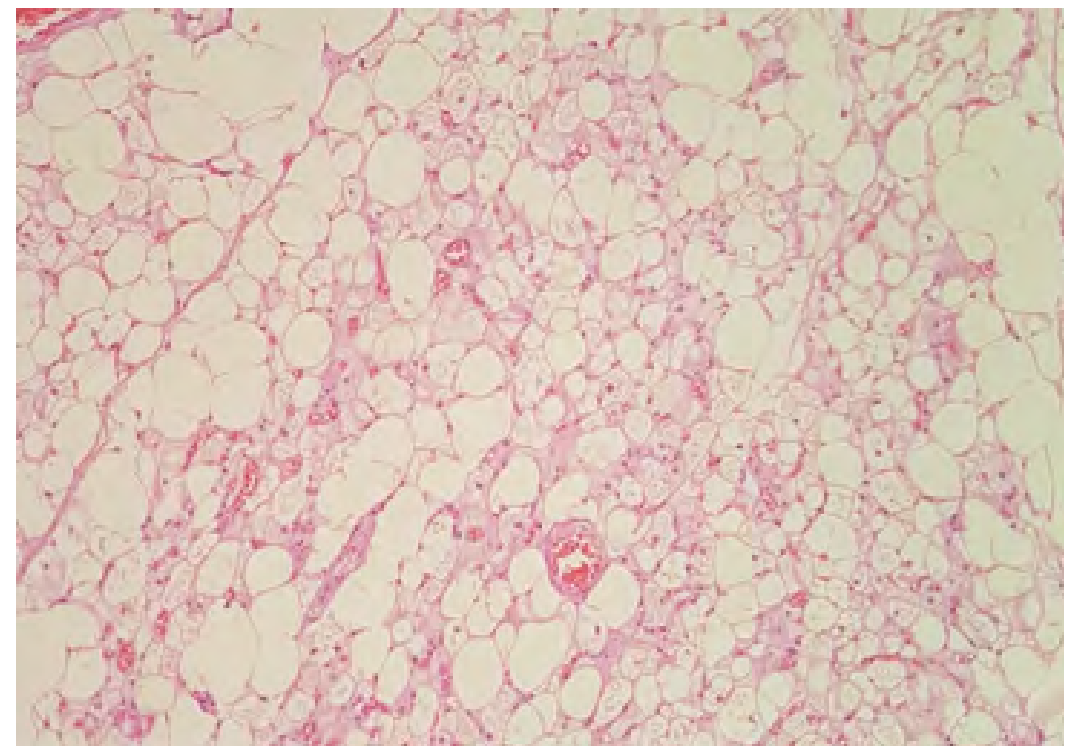

\section{Discusión}

El hibernoma es una neoplasia benigna de tejidos blandos que se origina en la grasa parda. Es un tumor de crecimiento lento y generalmente indoloro. Ocurre con mayor frecuencia en adultos entre la tercera y cuarta décadas de la vida y con una igual distribución en ambos sexos. Tienden a ser tumores grandes con un tamaño promedio de $9.3 \mathrm{~cm}$ (rango de 1-24 $\mathrm{cm}$ ) y muestran predilección por el tejido subcutáneo de localizaciones tales como el muslo (más frecuente), axila, cuello, mediastino y retroperitoneo. Otras localizaciones menos frecuentes son: cuero cabelludo, nalgas, fosa poplítea, zona perineoescrotal, intracraneal/ intraespinal, periureteral y cordón espermático. Únicamente el 10\% de los hibernomas son intramusculares. ${ }^{(1,2)}$
Dada la naturaleza benigna de este tumor, no presentan recurrencia tras un tratamiento quirúrgico ni tampoco se han descrito casos de metástasis. ${ }^{(2)}$ No obstante, es importante destacar que en base a sus características clínicas y radiológicas son comúnmente confundidos con otras lesiones de estirpe adipocitaria, principalmente con tumores lipomatosos atípicos (ALT)/liposarcomas bien diferenciados (WDL). Los ALT son tumores localmente agresivos de frecuente aparición en extremidades inferiores y que son abordables mediante resección quirúrgica, mientras que los WDL designan lesiones en localizaciones diferentes a extremidades (por ejemplo, retroperitoneo) que refieren una cirugía más radical para lograr márgenes libres de enfermedad. En un estudio 
retrospectivo en el que se analizaron las características clínico-patológicas de 64 casos de hibernoma confirmados histológicamente entre los años 2000 y 2017, se llegó al diagnóstico de ALT en el 66\% de los casos, aplicando radioterapia neoadyuvante en uno de los casos. Se precisa por tanto un buen diagnóstico diferencial para evitar aplicar tratamiento innecesario y potencialmente nocivo para el paciente. ${ }^{(2)}$

Clínicamente se caracterizan por ser lesiones únicas móviles, palpables, de crecimiento lento e indoloro y consistencia blanda. ${ }^{(1,3)}$ Los estudios de radiodiagnóstico recomendados para su estudio son la ecografía, TC, RM y la tomografía por emisión de positrones (PET-TC). La ecografía puede mostrar una lesión hiperecogénica o ecogénicamente heterogénea bien circunscrita o con menor frecuencia sin límites definidos, ${ }^{(3)}$ similar al caso descrito. El uso de Doppler revela en ocasiones hipervascularización intratumoral. En la TC aparecen como masas lobuladas bien definidas de densidad grasa, aunque ligeramente más hiperdensas que la grasa locorregional y con captación difusa de contraste. En lo que respecta a la RM, se caracterizan por presentar una señal hiperintensa, -ligeramente inferior a la grasa de tejido subcutáneo-, así como realce difuso de contraste, característica que diferencia a los hibernomas de los lipomas, que no muestran captación. ${ }^{(1)}$ La supresión incompleta del tejido graso en secuencias selectivas como SPAIR puede ser una pista útil para reconocer un hibernoma, aunque en otras como la practicada en nuestro caso (DIXON), con mayor capacidad supresora sí pueden hacerlo. ${ }^{(4)}$ La presencia de septos irregulares y gruesos ( $>2 \mathrm{~mm}$ ) y de elementos no grasos dentro del tumor pueden ser útiles para diferenciar los ALT/WDL de lipomas e hibernomas. ${ }^{(1)}$ Por último, el PET-TC es otra herramienta que puede ayudarnos a distinguir entre liposarcomas y tumores de tejido graso pardo, pues éstos generalmente poseen un valor estandarizado de captación máximo (SUVmax) elevado y superior al del WDL al contener células provistas de gran cantidad de mitocondrias. ${ }^{(5)}$ En el paciente que este artículo presenta, no llegó a realizarse esta prueba pues los hallazgos de la RM eran compatibles con el diagnóstico de sospecha. Sin embargo, puesto que ninguna prueba de imagen puede diagnosticar de forma inequívoca esta entidad, es precisa una confirmación anatomopatológica para alcanzar el diagnóstico definitivo, ${ }^{(1)}$ siendo posible obtener una biopsia de la lesión para su estudio. ${ }^{(3)}$ El tratamiento de elección es la resección completa tumoral. ${ }^{(1)}$

En el análisis macroscópico, los hibernomas se caracterizan por ser lesiones de aspecto graso, lobuladas y encapsuladas con un tono amarillo-marrón rojizo al corte. En lo que respecta al estudio microscópico, las células tumorales están organizadas en lóbulos separados por septos finos y se distinguen tres tipos celulares diferentes en proporciones variables: células de tipo lipoblasto, caracterizadas por presentar un núcleo pequeño citoplasma claro multivacuolado, adipocitos maduros y por último adipocitos del tejido graso pardo, que presentan un citoplasma eosinofílico granular.

Se han descrito cuatro variantes histológicas: clásico ( $~ 80 \%$ de los casos), mixoide ( $9 \%)$, lipoma-like (7\%) y de células fusiformes (2\%). ${ }^{(2)}$ Típicamente en los hibernomas no se localizan focos de mitosis o atipia celular, hallazgos que los diferencian de un ALT/WDL. No obstante, es preciso tener en cuenta que se han descrito casos de liposarcomas bien diferenciados en los que se han detectado áreas de hibernoma. ${ }^{(6)}$ 
Hibernoma perineal: presentación de un caso clínico y revisión de la literatura. Legido-Gómez Ó., et al.

Son de utilidad diagnóstica como marcadores de inmunohistoquímica los oncogenes $M D M-2, C D K-4$ y el gen regulador $p 16$, todos ellos implicados en el control del ciclo celular. Característicamente, los hibernomas no expresan ninguno de estos marcadores, al contrario que los ALT/WDL y liposarcomas desdiferenciados. ${ }^{(1,6)}$ Excepcionalmente podrían expresarse en áreas de hibernoma de un liposarcoma, en las que también existiría atipia y núcleos hipercromáticos. ${ }^{(6)}$ En cambio, la mayoría de hibernomas son positivos para la proteína S-100.(2) Es muy frecuente que los hibernomas muestren anormalidades en el cromosoma 11 (11q13) en forma de deleciones del gen MEN1 (neoplasia endocrina múltiple de tipo 1), siendo por tanto una manifestación a tener en cuenta en pacientes afectados por dicho síndrome. ${ }^{(7)}$

\section{Conclusión}

El hibernoma se trata de una rara neoplasia benigna de tejido graso pardo que con frecuencia se diagnostica erróneamente como lipoma o liposarcoma, por lo que es crucial tener en cuenta todos los elementos diferenciadores a nuestro alcance que nos permitan llegar al diagnóstico correcto y evitar tratamientos potencialmente iatrógenicos.

\section{Financiación}

No se recibió patrocinio de ningún tipo para llevar a cabo este artículo.

\section{Conflicto de intereses}

Los autores declaran no tener ningún conflicto de intereses.

\section{Referencias}

1. Patil SD, Sheik AR, Tewari V, Mutreja D. Hibernoma: A missed diagnosis!! Indian Journal of Pathology and Microbiology. 2019;62(3):461. doi: 10.4103/IJPM.IJPM_577_18

2. Al Hmada Y, Schaefer I-M, Fletcher CDM. Hibernoma Mimicking Atypical Lipomatous Tumor: 64 Cases of a Morphologically Distinct Subset. Am J Surg Pathol. 2018;42(7):951-7. doi: 10.1097/PAS.0000000000001061

3. Collado L, Sierre S, Bosalec A, Lipsich J. Hibernoma: tumor retroperitoneal de grasa parda: Informe de un caso pediátrico. Arch argent pediatr. 2011;109(6):126-9.

4. Kishida Y, Koyama H, Seki S, Yoshikawa T, Kyotani K, Okuaki T, et al. Comparison of fat suppression capability for chest MR imaging with Dixon, SPAIR and STIR techniques at 3 Tesla MR system. Magn Reson Imaging. 2018;47:89-96. doi: 10.1016/j.mri.2017.11.012

5. Pothen L, D'Abadie P, Kozyreff A, Mourin A, Coubeau L. Hibernoma mimicking liposarcoma. Lancet. 2018;392(10143):244. doi: 10.1016/ S0140-6736(18)31436-3

6. Hallin M, Schneider N, Thway K. Well-Differentiated Liposarcoma With Hibernoma-Like Morphology. Int J Surg Pathol. 2016;24(7):620-2. doi: $10.1177 / 1066896916653213$

7. Marchand L, Decaussin-Petrucci M, Giraud S, Cotton F, Thivolet C, Simon C. Hibernoma and multiple endocrine neoplasia type 1 syndrome: A non-fortuitous association? A case report and literature review. Ann Endocrinol (Paris). 2017;78(3):194-7. doi: 10.1016/j. ando.2017.03.001 